$10.2478 / \mathrm{gb}-2020-0008$

sciendo

\title{
Das Warten und die tote Zeit. Annäherung an Herta Müllers Kurzprosa Der Mensch ist ein großer Fasan auf der Welt.
}

\section{Béatrice-Kristine NICORIUC \\ Dr.; Cluj-Napoca; E-Mail: nbeatrice1@gmail.com}

\begin{abstract}
The paperdealswiththe rural environment in Herta Müller's Der Mensch ist ein großer Fasan auf der Welt. In the analysis the stress falls on the semantisation of the walkable space, like the countryside landscape and the built environment. This study also includes the presentation of interpersonal relationships of the village people and thematises the metaphorical spaces of oppression and fear, the metaphorical space of waiting.
\end{abstract}

Keywords: the terror of the surveillance state, the moral fall of the individual, fear.

Für die vorliegende Auseinandersetzung wird die Fassung von 2009 der Erzählung Der Mensch ist ein großer Fasan auf der Welt analysiert. Das Werk besteht aus 50 Kapiteln von sehr unterschiedlicher Länge. Manche sind nur eine halbe Seite, die anderen betragen 5 Seiten. Vom Inhalt her haben die Kapitel nicht immer eine direkte Verbindung zum Protagonisten Windisch, sondern beziehen sich auf das Dorfleben in verschiedenen Lebensereignissen, auf die unterschiedlichen Seiten des Landlebens, auf die Dorfbewohner, die immer eine unmittelbare Beziehung zur Hauptperson und zu dessen Umfeld haben. Die Verbindung zu Windisch ist im ganzen Werk präsent, die Verbindung ist Windisch selbst, der am dörflichen Geschehen meistens als Beobachter teilnimmt. Das letzte Kapitel grenzt sich von dem Rest ab, es ist titellos und beschreibt die Rückkehr der 
Windischs ins Dorf. Mit diesem losen Kapitel ist die Grenzüberschreitung plastisch dargestellt.

Meine Untersuchung zielt auf die Darstellung unterschiedlicher Motive, die das endlose Warten an der Grenze zwischen Bleiben und Ausreisen, zwischen Leben und Tod schildern.Der allgemeine Zustand ist das zerrende Warten, dessen Personifikation der Müller Windisch ist. Er ist die Hauptfigur der Erzählung, dessen Leben durch das eigene Scheitern und durch die einengende Dorfgemeinschaft charakterisiert wird. Die Todesangst und der Tod sind ständige Begleiter der Ereignisse, denen nicht nur Windisch, sondern alle Protagonisten der Erzählung ausgeliefert sind.

Das Dorf, das Herta Müller in der Erzählung Der Mensch ist ein großer Fasan auf der Welt beschreibt, ist ein hermetisch abgeschlossenes Milieu, wie eine große Schachtel ohne Fenster. In dieser Schachtel sind die einzelnen Menschen voneinander durch Wände und Fenster abgegrenzt, eingesperrt in ihr minderwertiges und auswegloses Leben. Zerfall, Schande, Angst und Tod sind die Schlüsselwörter, die über die ganze Erzählung das Dorfleben definieren. Die Protagonisten leben ihr eintöniges Leben von Tag zu Tag, Jahr zu Jahr. Das Wiederkehren der Ereignisse deutet auf die Unendlichkeit dieser Lebensform, aus der kein Entkommen in Sicht ist. Das Motiv der Grenze zieht über die ganze Erzählung in unterschiedlichen Formen: die Grenze zwischen Familienmitgliedern, zwischen Opfern und Tätern (Dorfbewohner und Machtleute), zwischen Ausreisen und Bleiben und schließlich die Grenze zwischen Leben und Tod.

\section{Räumliche Grenzmotive}

Windisch, der Müller, lebt auf dem Dorf und wartet auf den Reisepass, um Rumänien zu verlassen und nach Deutschland umzusiedeln. Sokönnteman in einem SatzdieAusgangssituation 
des Protagonisten schildern. Doch seine Lage ist viel komplizierter und traumatisierender, in einem Land, wo man keinen Reisepass besitzen darf und eine Ausreise aus dem Land von dem Geheimdienst, genannt Securitate, genehmigt werden soll. Windisch ist damit dem politischen Machtsystem ausgeliefert und soll sich unterwerfen. Das verlangen unterschiedliche Machtinhaber von ihm, wie der Milizmann, der Pfarrer, der Bürgermeister und der ganze staatliche Überwachungsapparat. So ist Windisch dem Warten ausgeliefert, er steht an der Demarkationslinie zwischen Bleiben-müssen und Weggehen-können. Das Warten bedeutet für Windisch den sicheren Tod, die Bewegung dagegen bedeutet ihm das Überleben, trotz ihrer kreisenden Art. Der Protagonist verläuft jeden Tag denselben Kreis im Dorf, das hält ihn am Leben. Die Wut, Frustration und Angst Gefühle die Windisch rotationsweise empfindet - werden mithilfe plastischer Sprachbilder auf die Außenwelt gelagert. Natur und Umgebung, also Pflaster, Straße, Mühle werden Träger der Gefühle, verräumlichen die Entfaltung dieser Gefühle:

Windisch hört ein Blatt auf dem Pflaster des Gangs. Es kratzt auf den Steinen. Die Wand ist lang und weiß. Windisch schließt die Augen. Er spürt, wie die Wand an sein Gesicht wächst. Der Kalk brennt an seiner Stirn. Ein Stein im Kalk öffnet das Maul. Der Apfelbaum zittert. Die Blätter sind Ohren. Sie horchen. Der Apfelbaum tränkt seine grünen Äpfel. ${ }^{1}$

Das Blatt ist in seiner natürlichen Form und Größe ein leichtes, zierliches und meist lautloses Objekt. Hier wird es überdimensioniert und mit lautem Geräusch und unangenehmem Gefühl assoziiert. Es kratzt auf dem Pflasterstein. Die Wand ist eine drohende, zerdrückende Sperre, die wächst ins Gesicht, drückt das Individuum nieder und lässt es verschwinden. Das Ich wird in der Sperre der Gesellschaft und der Umgebung aufgelöst, Mund und Ohren sind mit Kalk und Bäumen eins. Herta Müller

${ }^{1}$ Müller, Herta: Der Mensch ist ein großer Fasan auf der Welt. Frankfurt a.M. 2009, S. 31f. 
ist hier auch, wie in den anderen Prosawerken an den Menschen, als Teil eines Geflechts interessiert. Der Mensch ist keine geschlossene Einheit. ${ }^{2}$ Diese Osmose des Menschlichen mit den Objekten, Tieren und Pflanzen der Umgebung ist in den Müllerschen Werken immer präsent, und wird als Stilmittel für die Vergrößerung des Angstzustandes benutzt. Bäume erscheinen als Messer, Pflanzen am Wegrand stellen eine Bedrohung dar.

Die Auswanderung ist mit Angst vor dem Machtsystem durchtränkt, Windisch ist den Schikanen der Macht ausgeliefert, doch weigert sich völlig sich zu unterwerfen. Er befindet sich auf dem Spannungsfeld zwischen Kampf und Aufgeben. Die anfängliche Entschlossenheit „Mann über den Terror und Verhinderungen zu sein" ${ }^{33}$ wandelt sich schleichend in eine totale Resignation, er muss annehmen, dass er den Kampf gegen die Repräsentanten der Macht verloren hat. Er muss seine Tochter Amalie als Gegenware für die Ausreisegenehmigung einzahlen. Seine Frust und Wut lassen sich als Narbe in sein Gesicht hineinwachsen; die Narbe am Gesicht ist die äußere Materialisierung der inneren Narbe. Diese Narbe wird immer tiefer und verewigt sich während des Wartens: „Windisch hat viele Wochen lang unterm Aug eine Wunde. Sie ist rot. Sie hat einen weichen Rand aus Eiter. [...]»Vielleicht liegt die Kruste in der tiefen Stelle«, denkt Windisch. "“4 Die tiefe Stelle an der Mühle symbolisiert das ewige Wiederkehren an denselben Ort, die kreisende Bewegung, die Sperre. Die Narbe und die Kruste

${ }^{2}$ Vgl. Zur Kritik des homo clausus. Elias, Norbert / Scotson, John L.: The Established and the Outsiders. A Sociological Enquiryinto Community Problems. London, 1994.

3 „Der Mensch ist stark, sagt er, stärker als das Vieh.“ Müller, Herta: Der Mensch ist ein großer Fasan auf derWelt, S. 8. So formuliert Windisch seine Antwort auf das vom Nachtwächter postulierte Sprichwort Der Mensch ist ein großer Fasan auf der Welt. Windisch empfindet den Nachtwächter als minderwertig.

${ }^{4}$ Müller, Herta: Der Mensch ist ein großer Fasan auf der Welt, S. 24. 
sind die äußere Materialisierung des inneren Schmerzzustandes. Die tiefe Stelle ist ein semantisierter Raum der Seele, in der eine verkrustete Narbe wächst. Der Schmerz und die Angst sind schon seit langer Zeit Begleiter von Windisch. Dieses Bild ist aus unterschiedlichen Teilelementen zu einem Ganzen, semantischen Raum geformt, die Narbe an der Haut, die tiefe Stelle auf der Straße und der seelische Zustand formen gemeinsam den metaphorischen Raum der Wut und Frust.

\section{Das Fenstermotiv}

Der allgemeine Ort in der Erzählung, wie auch in anderen Werken Müllers, ist ein Ort des Dazwischens: zwischen den Welten, Wirklichkeiten und Räumen. ${ }^{5}$ Dieses Dazwischen-Sein materialisiert sich mehrfach im Fenster-Motiv, das sowohl trennend als auch verknüpfend wirkt. Der Blick des Protagonisten wird oft als ein Blick durch das Fenster dargestellt. Das Fenster lässt zwar, das wonach sich Windisch sehnt erblicken, doch erreichen kann er es nicht. Die Fensterscheibe wird zu einem semantischen Raum des Wartens auf die Ausreise und der Sehnsucht nach dem Unerreichbaren.

Die Angst und die Machtlosigkeit begleiten Windisch über die ganze Erzählung bis zur Ausreise. Sein Leben im Dorf ist als eine kreisende Bewegung dargestellt. Er widerlegt jeden Tag denselben Weg zur Mühle, durch das Dorf und zurück. Er hat mehrere Anhaltspunkte auf diesem Kreis, wo er manchmal stehenbleibt oder nicht, wie die tiefe Stelle auf dem Weg, das Haus des Tischlers oder des Kürschners oder das Kriegerdenkmal. Dieser Kreis-Weg ist das Leben für Windisch. Die Wiederholung desselben Weges, die kreisende Bewegung ändert diesen Raum, Windischs Wahrnehmung des kreisförmigen, gesperrten Raumes ändert sich auch, der Weg wird eng und bedrohlich. In der

5 Eke, Norbert Otto: Autoren. Oskar Pastior. In: Eke, Norbert Otto (Hg): Herta Müller Handbuch. Stuttgart 2017, S. 149. 
Wahrnehmung der Umgebung, in der die Angstbilder entstehen, ist Windisch meistens durch ein Fester oder einem Vorhang vom Wahrgenommenen getrennt. Windisch wird überproportional klein und die umgebenden Gegenstände werden groß und agieren meistens selbständig. Sie sind meistens mit Eigenschaften versehen, die ihnen unnatürlich sind - Blätter sind laut und tun einem weh, Räume stehen auf dem Kopf und beginnen ein eigenes Leben, die Messerschneiden lassen sich schwer kontrollieren, die Starre und die Unbeweglichkeit wirken drohend.

Das Fenster als Grenzmotiv kommt an vielen Stellen vor, es grenzt Windisch von den Dorfbewohnern ab. Windisch ist auf die Bewohner, die den Pass schon bekommen haben, neidisch. Beim Rasieren steht Windisch vor dem Fenster, er sieht dadurch das grüne Gras. ${ }^{6}$ Das frische Gras symbolisiert die Jahreswende, die Zeit ist vergangen und er steht immer noch vor seinem alten Spiegel, in seinem alten Haus, im selben Dorf. Das Motiv des Fensters symbolisiert Windischs Neid und die damit verbundene Distanz, die immer größer wird zwischen ihm und den anderen auch zu seiner Familie. Eine der bedeutendsten Szenen für Windischs traumatisierten Wahrnehmung des Raumes und das krankhafte Verlangen für die Ausreise, ist die vor dem Haus des Kürschners. Windisch beobachtet das Zimmer des Kürschners, von außen, durch das Fenster „Hinterm Apfelbaum hängen die Fenster des Kürschners. Sie sind hell beleuchtet. »Der hat den Pass «, denkt Windisch. Die Fenster sind grell, und nackt ist das Glas. Der Kürschner hat alles verkauft. Die Zimmer sind leer. »Die haben die Vorhänge verkauft«, sagt Windisch vor sich hin. ${ }^{67}$

Das Fenster wird hier mit doppelter Konnotation versehen. Für Windisch bedeutet es wiederum eine Trennwand zum Kürschner, für den Kürschner aber ist es die Öffnung. Die Wörter hell, grell und nackt weisen auf den offenen Charakter

${ }^{6}$ Müller, Herta: Der Mensch ist ein großer Fasan auf der Welt, S. 24.

7 Ebd., S. 18. 
des Fensters. Das nackte Fensterglas und die Abwesenheit der Vorhänge bedeuten für Windisch, dass der Kürschner den Pass bekommen hat. Das Zimmer ist schon für die Ausreise vorbereitet, die Wände sind leer, Koffer stehen herum. Neid und Frust überwältigen Windisch bei dem Anblick der Familie des Kürschners. Die Wut, die ihm beim Zuschauen packt, löst bei Windisch eine Halluzination vor, er nimmt das Zimmer des Kürschners als in einem Traum wahr. In seiner Wahnvorstellung gibt es keinen Unterschied zwischen seiner Raumperzeption und der Raumkonstitution. ${ }^{8}$ Schwarze Flecken dominieren seine Wahnvorstellung, der Raum wird gedreht, der Fußboden dreht sich, tauscht seinen Platz mit der Decke, die zu liquiden Gegenständen werden und aneinander schwemmen. Vor dem Fenster erlebt Windisch als Zuschauer eine Erschütterung, ein Erdbeben in sich selbst, betrunken von Neid und Frustration und vom neurotischen Druck ausreisen zu wollen und nicht können:

Der Fußboden ist schief. Der Fußboden hebt sich. Er steigt hoch an der Wand. Er stellt sich vor der Tür. Der Kürschner zählt das zweite Bündel Geld. Der Fußboden wird ihn verdecken. Die Frau des Kürschners bläst von der grauen Pelzkappe den Staub. Der Fußboden wird sie zur Decke heben. Neben dem Kachelofen hat die Wanduhr einen langen weißen Fleck geschlagen. Neben dem Kachelofen hängt die Zeit. Windisch schließt die Augen. »Die Zeit ist zu Ende«, denkt Windisch. Er hört den weißen Fleck der Wanduhr ticken und sieht das Zifferblatt aus schwarzen Flecken. Ohne Zeiger ist die Zeit. Nur die schwarzen Flecken drehen sich. Sie drängen sich. Sie drücken sich aus diesem weißen Fleck hinaus. Fallen der Wand entlang. Sie sind der Fußboden. Die schwarzen Flecken sind der Fußboden im andren Zimmer. ${ }^{9}$

Hinter dem Fenster, in Windischs subjektive Wahrnehmung, wird die Welt auf dem Kopf gestellt und zum Hindernis verwandelt. Der Fußboden hindert den Kürschner das Haus zu verlassen, der Fußboden verliert seine bisherige Funktion, wird

${ }^{8}$ Löw, Martina: Raumsoziologie. Frankfurt a.M. 2001, S. 164.

9 Müller, Herta: Der Mensch ist ein großer Fasan auf der Welt, S. 19. 
zur Decke und zum Hindernis. Man befindet sich in einer Zeitlosigkeit, die Welt dreht sich und möchte damit auch die Chronologie aufbrechen. Die Uhr erscheint ohne Zeiger, ein wiederkehrendes Motiv, auch in Reisende auf einem Bein gibt es die Uhr ohne Zeiger. Klang und Sicht fließen ineinander, er hört den Fleck ticken. Diese räumlich und zeitlich metaphorisierten Hindernisse stellen ihm die Ausreise als unmöglich dar. Den Riss unter den Füßen und die Erschütterung spürt er auch im letzten Kapitel: „Der Gehsteig ist rissig. [...] In der tiefen Stelle spürt Windisch das Ziehen der Risse unterm Schuh. " ${ }^{\text {"10 }}$ Das Ziehen der Risse steht für Windischs Machtlosigkeit, er ist nicht in der Lage von diesem Bodenwegzukommen. Die Trägheit, die Frust und der Wut versetzen ihn in einen allgemeinen Angstzustand.Der Tod und die Todesangst finden ihre semantischen Räume in Gegenständen und Naturelementen wieder. Die Landschaft und die Elemente der mittelbaren Umgebung werden zu metaphorischen Räumen der Angst und des Todes.

\section{Der Tod und die tote Zeit}

Windisch fährt jeden Tag in die Mühle, durch das Dorf. Die Straße führt am Kriegerdenkmal vorbei, das meistens im Nebel steht. Er zählt die Tage, bis er seinen Reisepass bekommt. Seine Fahrt wiederholt er jeden Tag seit zwei Jahren, er zählt auch die Jahre: „Windisch zählt zwei Jahre vor dem Kriegerdenkmal und zweihunderteinundzwanzig Tage in der tiefen Stelle vor der Pappel. " ${ }^{11}$ Er kehrt jeden Tag zur selben Stelle zurück, bleibt stehen, womit seine Frust und der Wille auszureisen steigt. Doch die Fahrt, der Weg führt ihn wieder zum Ausgangspunkt, das Dorf ist metaphorisch ein gesperrter Ort, kein Weg, den Windisch geht, führt aus dem Dorf hinaus. Windisch erlebt jeden Tag eine Reise im eigenen Dorf. An einem Abend steigt er von seinem

\footnotetext{
${ }^{10}$ Ebd, S. 109.

${ }^{11}$ Ebd, S. 5.
} 
Fahrrad vor der Kirche ab, wo die Gestalt der Heiligen Antonius ihm als eingesperrt erscheint. Der Heilige Antonius als Schutzpatrone - unter anderen - der Reisenden, ${ }^{12}$ konnte ihm eine Befreiung bedeuten, doch Windisch bleibt vergebens an der Statue stehen.

Windischs Welt ist im Untergang begriffen, die umgebenden Ereignisse sind alle mit dem Tod und Vergehen in Verbindung, doch ist das Vergehen der Zeit ein sehr relatives Phänomen. Der Tod, das Verwelken, das Weggehen deuten auf den zeitlichen Ablauf. Bei anderen Tätigkeiten und Geschehnissen erscheint die Zeit als stillstehende Masse, das beweisen die vielen Momentaufnahmen, die keine zeitliche Linearität aufweisen, sondern nur nebeneinander gereiht sind. Das Gesamtbild ist auf kleine Bildschnitte aufgeteilt, damit ist keine zeitliche Kontinuität der Ereignisse aufzufinden. Die Linearität zu finden bleibt dem Leser überlassen.

Die Linearität in der Gesamterzählung wird immer durch das endlose Warten unterbrochen, es gibt keinen Expliziten Anfang oder Ende. Symbole, die auf das zeitliche Vergehen verweisen sollen, sind ihren Konnotationen entlastet, Flüsse fließen nicht, die Uhr hat keine Zeiger, oder man sieht nur die Stelle, wo sie an der Wand gehängt war. Die ,parataktische Montage von Bildern [führt zur] Enttemporalisierung oder

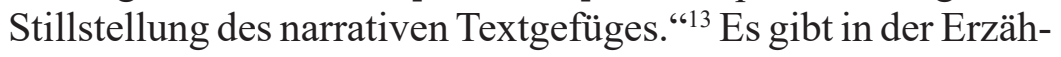
lung zwei konkrete Deutungen auf das Zeitvergehen. Einerseits sind es die Jahreszeitenänderungen, die man begleitend zu den Gesprächen zwischen Windisch und dem Nachtwächter observieren kann und andererseits an den wiederholten Fahrten zur

${ }^{12}$ Schlagwort Antonius von Padua. In: https://www.heiligenlexikon.de/ BiographienA. Zugriff: 28.04.2020.

${ }^{13}$ Roberg, Thomas: Bildlichkeit und verschwiegener Sinn in Herta Müllers Erzählung Der Mensch ist ein großer Fasan auf der Welt. In: Köhnen, Ralph (Hg.): Der Druck der Erfahrung treibt die Sprache in die Dichtung. Bildlichkeit in Texten Herta Müllers. Frankfurt a. M 1997, S.39. 
Mühle. Die Natur ändert sich, das Jahr vergeht und das Leben der Dorfbewohner bleibt dasselbe eintönige Leben.

Die tote Zeit zeigt sich auch in den Gesprächen, die Windisch mit dem Nachtwächter führt. Schweigen und Sprechen wechseln sich in diesen Dialogen. Wenn sie sprechen, vergeht ein wenig Zeit, wenn sie schweigen, ist die Zeit tot. Sprechen hat Lebendigkeit in sich, Schweigen ist Träger des Todes. Die Gespräche zwischen Windisch und dem Nachtwächter finden in der Nacht statt, die umgebende Natur weist auf Stille, nichts rührt sich. Die Mühle erscheint als stummer Riese, alles ist dunkel und ruhig, der Nachtwächter schläft. Windisch und auch die anderen Protagonisten benutzen das Schweigen als Schutz vor der Abhörbarkeit. In den Gesprächen zwischen Windisch und dem Nachtwächter kommt es oft zum Schweigen, die Umgebung der Mühle, das Wasser und andere Gegenstände überliefern das Schweigen. „Die Mühle ist stumm. Stumm sind die Wände und stumm ist das Dach. Und die Räder sind stumm." "14 Auf das Schweigen deuten auch die eintönigen Blumen und ihr verwelkter Zustand, das Rosengestrüpp hat kahle Dornen, das Unkraut ist rostig.

Der Tod kommt obsessiv in allen Geschehnissen und Gegenständen vor, das Weggehen und das Verlassen deuten alle in die Richtung des Todes. Doch Windisch wehrt sich gegen den Tod und die Erniedrigung, indem er seine Macht gegenüber dem Nachtwächter ausübt. Windisch empfindet den Nachtwächter als dumm und unfähig seinen Zustand zu ändern. Doch diese Konstellation, die Windisch zwischen sich und dem Nachtwächter aufstellt, wird sich am Ende der Erzählung ändern.

In der Außenwelt, die Windischs inneres Zeitempfinden widerspiegelt, scheint die Zeit still zu stehen, Windisch zählt die Tage, die Stunden, die Monate. Er bildet sich ein, Herr über die Zeit zu sein, sie zum Vergehen bezwingen zu können. Das existiert nur in seinem Kopf und die Liquidität des Vergehens ist

${ }^{14}$ Müller, Herta: Der Mensch ist ein großer Fasan auf der Welt, S. 6. 
auch Produkt seiner Imagination und unterliegt einer zeitlichen Simultanität. ${ }^{15}$ Er ist derjenige, der seine Zeit bestimmen kann, seine innere Zeit, die aber mit der Äußeren nicht korrespondiert. Insofern ist sie liquid, die Zeit verrinnt in der von Windisch bestimmten Geschwindigkeit: „Windisch hört seinen Schädel klopfen und denkt: Mein Kopf ist eine Uhr. Er steckt die Schlüssel in die Tasche. Der Hund bellt. Ich werde sie aufziehen, bis die Feder reißt, sagt Windisch laut.“16

Draußen ist das Warten erwünscht, eine Unterwerfung der Zeit und den Mächtigen gleicherweise. Die Federn seiner inneren Uhr sind schon am Reißen, die Ungeduld wird bedrückend. Damit setzt er sich dem Tod gegenüber, er will ihn beherrschen, doch am Ende muss er sich völlig erniedrigen lassen, um an den Pass zu kommen.

Das ganze Dorf erscheint aus der Zeit gefallen zu sein, es geschieht eine Entzeitlichung des Raumes, ${ }^{17}$ somit gerät das Dorf in eine mythisierte Lage, es wird zum fabelhaften Ort, mit dem Requisiten einer Phantasiewelt, wie der Apfelbaum, der seine eigene Früchte verzehrt, die tötende Dahlie und die den Tod verkündende Vögel - die Eule und die Drossel. Die Eule, der Protagonist der Märchenwelt ist in der gesamten Erzählung als Leitmotiv anwesend, zieht den schwarzen Faden des Todes über das Dorf. Sie ist nicht nur Symbol des Todes, sondern sie bestimmt wer zunächst sterben wird, indem sie sich auf das Dach des Hauses setzt, wo jemand sterben sollte. Die Eule erscheint in einer Nacht über dem Teich an der Mühle. Die alte Kroner liegt seit mehreren Tagen im Sterbebett, doch die Eule setzt sich nicht auf ihr Dach: „Bei der alten Kroner brennt seit

\footnotetext{
${ }^{15}$ Bronfen, Elisabeth: Der literarische Raum. Tübingen 1986, S. 7.

${ }^{16}$ Müller, Herta: Der Mensch ist ein großer Fasan auf der Welt, S. 7.

${ }^{17}$ Eke, Norbert Otto: „Überall, wo man den Tod gesehen hat“. Zeitlichkeit und Tod in der Prosa Herta Müllers. Anmerkungen zu einem Motivzusammenhang. In: Eke, Norbert Otto (Hg.): Die erfundene Wahrnehmung. Annäherung an Herta Müller. Paderborn 1991, S. 87.
} 
drei Nächten das Licht. Windisch schiebt das Fahrrad. Sie kann nicht sterben, sagt er, die Eule setzt sich auf kein Dach." ${ }^{\text {"18 }}$ Der Tod wäre in diesem Fall eine Befreiung, doch die Eule ist mächtiger, die Erlösung, die sie der alten Kroner erbringen könnte, bleibt aus. Das endlose Warten wird hier auch metaphorisch im Bild der Eule dargestellt, die alte Kroner soll auf ihren Tod warten. Sie erscheint am Fenster des Tischlers sogar verdoppelt, ihre Macht wird verdoppelt, so wird es dem beobachtenden Windisch klar, dass die alte Kroner gestorben ist. Als die Eule am Haus vorbeifliegt, nimmt Windisch den Sack auf seinem Fahrrad wie einen Toten wahr. Das Bild der Eule, der Ansicht des Sarges durch das Fenster erbringen Todesgefühle bei Windisch selbst. Der Tod gleitet nach Außen: „Der Sack ist feucht. Er hängt wie ein Schlafender über dem hinteren Rad. Wie ein Toter, denkt Windisch, hängt der Sack hinter mir." ${ }^{\text {"19 }}$ Das bedeutet auch eine Bedrohung für Windisch, der Tod sei hinter ihm, er folge ihm und es gibt kein Entkommen.

Der Sarg und der Sack stehen beide als allgemeingültige Symbole des Todes, die Behälter, die den Leichnam verhüllen. Windisch versucht dem Tod und der Erniedrigung zu widerstehen, ein Widerstand gegen den Tod zeigt sich seiner elementaren und triebhaften Gestalt, der meistens durch Wut manifestiert. Dieser Kampf gegen den Tod und das System ist nie vernünftig, es kommt aus der tiefsten, triebhaften Inneren. Das Motiv des Sackes kommt in der Erzählung oft vor, etwa als Windisch das Mehl in Säcken zum Bürgermeister oder anderen Machtrepräsentanten trägt. Das Tragen der Säcke hat kein Ende, die Bestechung für den Pass ist nie genug. Beim Tragen der Säcke hört Windisch den Wind wie eine Nähmaschine, er nimmt es wahr, als würde der Wind einen Sack in die Erde nähen. Das Tragen und das Warten verwandeln den Sack in einen Sarg, die unendliche Bezahlung macht den Sack zum Behälter des Todes.

${ }^{18}$ Müller, Herta: Der Mensch ist ein großer Fasan auf der Welt, S. 10. ${ }^{19}$ Ebd, S. 12. 
Der Sack ist mehr als ein Element aus der Menge der Gegenstände, sondern er wird zum gelebten Raum des Todes. ${ }^{20}$

Ein Abbruch mit der drückenden, überwachenden Eigenschaft des Dorfes gelingt den Protagonisten nicht. Damit sind das Weggehen und das Ankommen per se weder gut noch schlecht, keine bedeutet eine echte Lösung für die existenzielle Not. Die Dorfgemeinschaft verschließt sich jeder Außeneinwirkung, den Figuren gelingt keinerlei Ausbruch aus diesem Kreis. Das einzige, das die Dorfgemeinschaft einen Zusammenhalt sichert, ist die gegenseitige Bewachung.

Die Analyse des Werkes Der Mensch ist ein großer Fasan auf der Welt hat gezeigt, dass das Dorf kein Ort der Harmonie und der Geborgenheit ist, in der sich der Mensch geborgen fühlt, sondern eine Zwangsgemeinschaft, die durch den Tod zusammengehalten ist.

\section{Literatur}

\section{Primärliteratur:}

Müller, Herta: Der Mensch ist ein großer Fasan auf der Welt. Frankfurt a. M. 2009.

Müller, Herta: Reisende auf einem Bein. Frankfurt a. M. 2010.

\section{Sekundärliteratur:}

Bronfen, Elisabeth: Der literarische Raum. Tübingen 1986

Dürckheim, Graf Karlfried von: Untersuchungen zum gelebten Raum. Erlebniswirklichkeit und ihr Verständnis. Systematische Untersuchungen II. In: Krueger, Felix (Hg.): Neue Psychologische Studien, 6. München 1932.

${ }^{20}$ Dürckheim, Graf Karlfried von: Untersuchungen zum gelebten Raum. Erlebniswirklichkeit und ihr Verständnis. Systematische Untersuchungen II. In: Krueger, Felix (Hg.): Neue Psychologische Studien, 6. München 1932, S. 390. 
Eke, Norbert Otto: ,, Überall, wo man den Tod gesehen hat“. Zeitlichkeit und Tod in der Prosa Herta Müllers. Anmerkungen zu einem Motivzusammenhang. In: Eke, Norbert Otto (Hg.): Die erfundene Wahrnehmung. Annäherung an Herta Müller. Paderborn 1991.

Eke, Norbert Otto: Autoren. Oskar Pastior. In: Eke, Norbert Otto (Hg): Herta Müller Handbuch. Stuttgart 2017.

Elias, Norbert / Scotson, John L.: The Established and the Outsiders. A Sociological Enquiryinto Community Problems. London, 1994.

Löw, Martina: Raumsoziologie. Frankfurt a.M. 2001.

Roberg, Thomas: Bildlichkeit und verschwiegener Sinn in Herta Müllers Erzählung Der Mensch ist ein großer Fasan auf der Welt. In: Köhnen, Ralph (Hg.): Der Druck der Erfahrung treibt die Sprache in die Dichtung. Bildlichkeit in Texten Herta Müllers. Frankfurt a. M 1997.

\section{Internetquelle:}

Schlagwort Antonius von Padua. In: https://www.heiligenlexikon.de/BiographienA. Zugriff: 28.4.2020. 